\title{
Tomographic 3D-PIV and Applications
}

\author{
Gerrit E. Elsinga ${ }^{1}$, Bernhard Wieneke ${ }^{2}$, Fulvio Scarano ${ }^{1}$,
} and Andreas Schröder ${ }^{3}$

1 Department of Aerospace Engineering, TU-Delft, The Netherlands, \{g.e.elsinga,f.scarano\}@tudelft.nl

2 LaVision GmbH, Göttingen, Germany, bwieneke@lavision.nl

3 Institut f. Aerodynamik und Strömungstechnik, DLR Göttingen, Germany, andreas.schroeder@dlr.de

Abstract. Tomographic particle image velocimetry is a 3D PIV technique based on the illumination, recording, reconstruction and analysis of tracer-particle motion within a three-dimensional measurement volume. The recently developed technique makes use of several simultaneous views of the illuminated particles, typically 4 , and their three-dimensional reconstruction as a light-intensity distribution by means of optical tomography. The reconstruction is performed with the MART algorithm (multiplicative algebraic reconstruction technique), yielding a 3D distribution of light intensity discretized over an array of voxels. The reconstructed tomogram pair is then analyzed by means of 3D crosscorrelation with an iterative multigrid volume-deformation technique, returning the three-component velocity vector distribution over the measurement volume. The implementation of the tomographic technique in time-resolved mode by means of high repetition rate PIV hardware has the capability to yield $4 \mathrm{D}$ velocity information. The first part of the chapter describes the operation principles and gives a detailed assessment of the tomographic reconstruction algorithm performance based upon a computer-simulated experiment. The second part of the chapter proposes four applications on two flow cases: 1. the transitional wake behind a circular cylinder; 2 . the turbulent boundary layer developing over a flat plate. For the first case, experiments in air at $\operatorname{Re}_{\mathrm{D}}=2700$ are described together with the experimental assessment of the tomographic reconstruction accuracy. In this experiment a direct comparison is made between the results obtained by tomographic PIV and stereo-PIV. Experiments conducted in a water facility on the cylinder wake shows the extension of the technique to timeresolved measurements in water at $R_{D}=540$ by means of a low repetition rate PIV system. A high data yield is obtained using high-resolution cameras $(2 \mathrm{k} \times 2 \mathrm{k}$ pixels) returning $650 \mathrm{k}$ vectors per volume. Measurements of the turbulent boundary layer in air at $\operatorname{Re}_{\theta}=1900$ provide a clear visualization of streamwise-aligned low-speed regions as well as hairpin vortices grouped into packets. Finally, in similar flow conditions the boundary layer is measured using a high repetition rate PIV system at $5 \mathrm{kHz}$, where the spatiotemporal evolution of the flow structures is visualized revealing a mechanism for the rapid growth of a Q2 event, possibly associated to the generation of hairpin-like structures. 


\section{Introduction}

The measurement of the instantaneous three-dimensional velocity field is of great importance in fluid mechanics and particularly in turbulence research since it reveals the complete topology of turbulent coherent structures. Moreover, three-dimensional measurements are advantageous for those situations where the flow does not exhibit specific symmetry planes or axes. The advent of PIV and its developments (stereo-PIV, [1], dual-plane stereo-PIV, [2]) showed the capability of the PIV technique to quantitatively visualize complex flows.

Several methods have been proposed to extend the PIV technique to a three-dimensional measurement technique. Scanning lightsheet [3], holography [4], 3D PTV [5] and very recently tomographic particle image velocimetry (tomographic-PIV, [6]) are the most popular approaches (see Arroyo and Hinsch in the present book for a complete review). In particular, the development of the tomographic-PIV technique was motivated by the need for a simple optical arrangement such as those used in the photogrammetric approach combined with a robust particle-volume reconstruction procedure not relying upon particle identification as opposed to the particle-tracking techniques. The latter is achieved by means of optical tomography. The resulting procedure offers the capability to handle relatively high seeding densities without compromising the robustness of the measurement.

The present work describes the working principle of tomographic PIV including a numerical assessment of its performances. The application to real experiments has two objectives: first, the method's accuracy can be assessed under actual experimental conditions by a comparison with stereoPIV; and secondly, the applications to the turbulent cylinder wake and the turbulent boundary layer directly show the type of flow information that can be obtained. Finally, the extension of the technique to time-resolved measurements (4D) in air and water flows is discussed and two applications are presented showing the time evolution of the 3D Karman wake behind a circular cylinder in water and the development of hairpin-like structures in a turbulent boundary layer.

\section{Principles of Tomographic PIV}

The working principle of tomographic PIV is schematically represented in Fig. 1. Tracer particles immersed in the flow are illuminated by a pulsed light source within a three-dimensional region of space. The scattered light pattern is recorded simultaneously from several viewing directions using CCD cameras. The Scheimpflug condition between the image plane, lens plane and the midobject plane is fulfilled by means of camera-lens adapters with two degrees of freedom. The particles within the entire volume are imaged in 
focus, which requires a relatively small lens aperture. The $3 \mathrm{D}$ particle distribution (the object) is reconstructed as a 3D light-intensity distribution from its projections on the CCD arrays. This reconstruction problem is of inverse nature and in general underdetermined, meaning that a single set of projections (viz. images) can result from many different 3D objects. Determining the most likely $3 \mathrm{D}$ distribution is the topic of tomography [7], which is addressed in the following section. Then, the particle displacement (hence velocity) within a chosen interrogation volume is obtained by the $3 \mathrm{D}$ crosscorrelation of the reconstructed particle distribution at the two exposures. The crosscorrelation algorithm is based on the crosscorrelation analysis with the iterative multigrid window (volume) deformation technique (WIDIM, [8]) extended to three dimensions.

The relation between image (projection) coordinates and the physical space (the reconstruction volume) is established by a calibration procedure similar to what is used in stereoscopic PIV or 3D-PTV. Each camera records images of a calibration target at several positions in depth throughout the volume, from which the calibration procedure returns the viewing directions and field of view. The tomographic reconstruction relies on accurate triangulation of the views from the different cameras. In particular, to successfully reconstruct particle images the accuracy requirement for the calibration is a fraction of the particle image size (typically below 0.4 pixels according to [6]). An adequate mapping function from physical space to the image coordinate is provided by either a pinhole model [9] or third-or-higher-order polynomials in $x$ and $y$ and linear along $z$ [10]. Procedures developed for the a-posteriori correction of calibration errors (self-calibration, [11]) have been shown to further increase the robustness of the reconstruction with respect to experimental uncertainties of the optical system (i.e., relative camera position).

\subsection{Tomographic Reconstruction Algorithm}

The novel aspect introduced with the tomographic-PIV technique is the reconstruction of the 3D particle distribution by tomography, which therefore deserves discussion in some more detail. Before the 3D intensity distribution can be reconstructed it is necessary to produce a linear mathematical model of the imaging system. In the present model (Fig. 2) the measurement volume is discretized as a 3D array of cubic voxel elements with physical coordinates $(X, Y, Z)$. The voxel intensity distribution is then defined as $E(X, Y, Z)$. Then, the projection of this volume intensity $E(X, Y, Z)$ onto the $i$ th image pixel with image coordinates $\left(x_{i}, y_{i}\right)$ returns the pixel intensity $I\left(x_{i}, y_{i}\right)$, which is known from the recorded images. The relation between the $3 \mathrm{D}$ light intensity and the image intensity is written in the form of a linear equation as:

$$
\sum_{j \in N_{i}} w_{i, j} E\left(X_{j}, Y_{j}, Z_{j}\right)=I\left(x_{i}, y_{i}\right),
$$




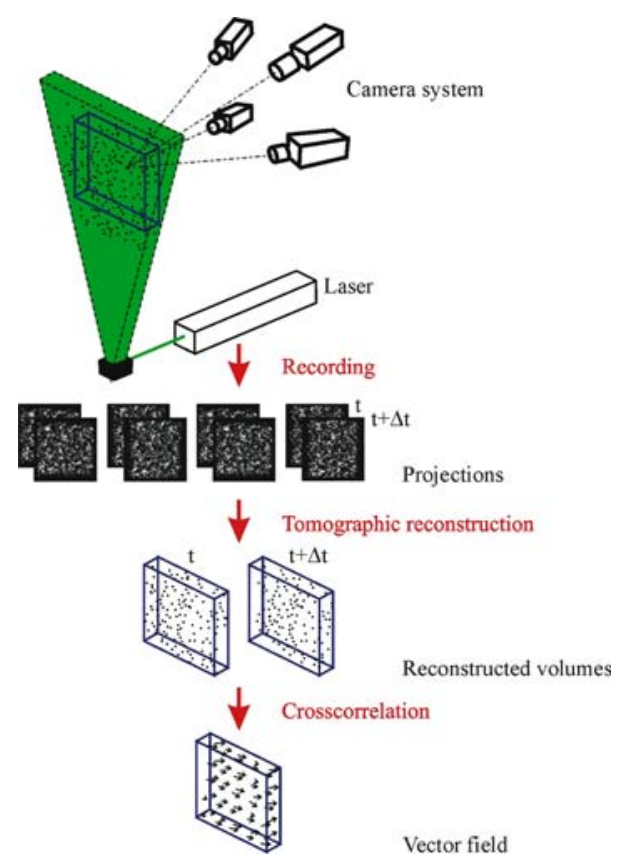

Fig. 1. Principle of tomographic PIV

where $N_{i}$ is the voxels neighborhood (typically a cylinder of $3 \times 3$ voxels cross section) around the line of sight of the $i$ th pixel $\left(x_{i}, y_{i}\right)$ through the volume. The weighting coefficient $w_{i, j}$ describes the contribution of the $j$ th voxel with intensity $E\left(X_{j}, Y_{j}, Z_{j}\right)$ to the pixel intensity $I\left(x_{i}, y_{i}\right)$ and is calculated as the intersecting volume between the voxel and the line of sight (having the crosssectional area of the pixel) normalized with the voxel volume. A unit ratio between the size of the pixel projection and the voxel element is assumed for simplicity, however, different values may be allowed. In particular, values lower than one have the advantage of reducing the size of the discretized volume for reconstruction and interrogation.

The iterative tomographic reconstruction algorithm MART (multiplicative algebraic reconstruction technique, [7]) is used to solve the system of (1). Starting from an initial guess $\left(E(X, Y, Z)^{0}\right.$ uniform and nonzero) the object $E(X, Y, Z)$ is updated at each full iteration in a loop over each pixel $i$ from all cameras and each voxel $j$ as:

$$
\begin{aligned}
& E\left(X_{j}, Y_{j}, Z_{j}\right)^{k+1} \\
& \quad=E\left(X_{j}, Y_{j}, Z_{j}\right)^{k}\left(I\left(x_{i}, y_{i}\right) / \sum_{j \in N_{i}} w_{i, j} E\left(X_{j}, Y_{j}, Z_{j}\right)^{k}\right)^{\mu w_{i, j}},
\end{aligned}
$$

where $\mu \leq 1$ is a scalar relaxation parameter (usually set to 1 for fastest convergence). The magnitude of the update is determined by the ratio of the measured pixel intensity $I$ with the projection of the current object 


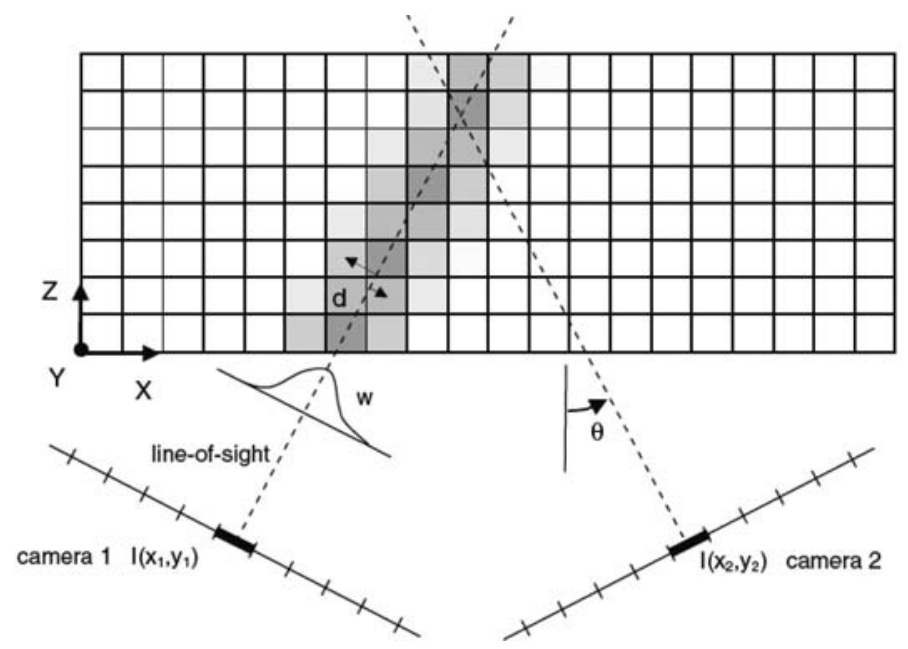

Fig. 2. Representation of the imaging model used for tomographic reconstruction. In this top view the image plane is shown as a line of pixel elements and the measurement volume is a $2 \mathrm{D}$ array of voxels. The gray level indicates the value of the weighting coefficient $\left(w_{i, j}\right)$ in each of the voxels with respect to the pixel $I\left(x_{1}, y_{1}\right)$

$\sum_{j \in N_{i}} w_{i, j} E\left(X_{j}, Y_{j}, Z_{j}\right)^{k}$. The exponent again ensures that only the elements in $E(X, Y, Z)$ affecting the $i$ th pixel are updated. Furthermore, the multiplicative MART scheme requires that $E$ and $I$ are positive-definite.

The iterative process has a relatively rapid convergence and can be stopped after 4 to 5 iterations without significant loss of accuracy. This is particularly interesting since the reconstruction procedure is computationally intensive. Moreover, experimental results show that additional iterations do not yield any significant change in the measured velocity vector field.

\subsection{Numerical Assessment of Performances}

The performance of the tomographic method was assessed by numerical simulations. Details are given in [6]. A domain with reduced dimensionality was adopted to simplify the computation and to ease the evaluation of results. Therefore, the $3 \mathrm{D}$ volume was reduced into a $2 \mathrm{D}$ slice and the images from the 2D CCDs were replaced by intensity profiles as issued by linear CCD arrays. This situation corresponds to that displayed in Fig. 2. A 2D particle field in the coordinate system $(X, Z)$ of $50 \times 10 \mathrm{~mm}^{2}$ is recorded by linear array cameras with 1000 pixels each. However, the qualitative information obtained by this procedure can be directly applied to the case of a 3D domain imaged by $2 \mathrm{D}$ images.

Figure 3 presents a detail of a reconstructed particle field by the MART algorithm. Distinct particles are returned at the correct position with an 

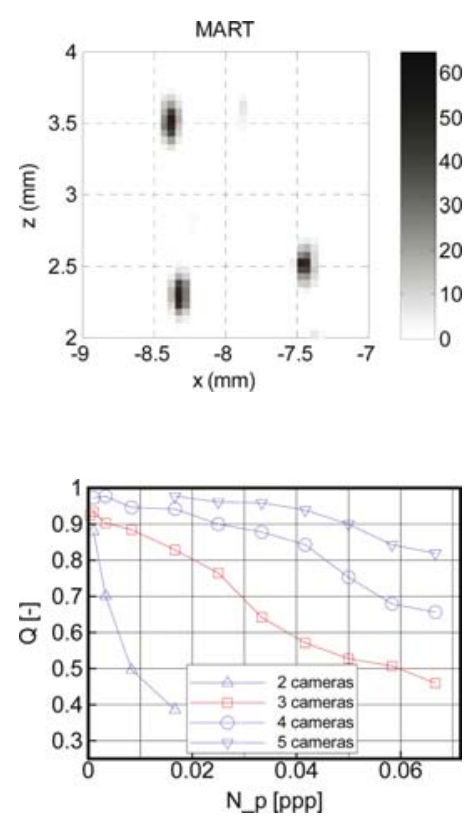

Fig. 3. Detail of the MART reconstruction showing individual particles. Circles indicate actual particle positions

Fig. 4. Reconstruction quality $Q$ as a function of number of cameras and particle image density

intensity distribution slightly elongated in depth depending on the viewing directions of the cameras. The reconstruction also contains a number of false intensity peaks ("ghost particles"), which do not correspond to actual tracers (e.g., $x=-7.8, y=3.6$ in Fig. 3). They occur when the particles cannot be located unambiguously inside the volume [5, 12], and their number increases with the number of actual particles, the particle image diameter and the depth of the measurement volume. However, as is also clear from the figure, the intensity of the ghost particles is low when compared to that of the actual particles and therefore their effect on the crosscorrelation peak and the velocity measurement is limited.

To quantify the relation between the accuracy of the tomographic reconstruction and experimental parameters, the reconstructed object $E_{1}(X, Z)$ is compared with the exact distribution of light intensity $E_{0}(X, Z)$ where the particles have been modeled by a Gaussian intensity distribution. The reconstruction quality $Q$ is defined as the normalized correlation coefficient of the exact and reconstructed intensity distribution according to:

$$
Q=\frac{\sum_{X, Z} E_{1}(X, Z) \cdot E_{0}(X, Z)}{\sqrt{\sum_{X, Z} E_{1}^{2}(X, Z) \cdot \sum_{X, Z} E_{0}^{2}(X, Z)}} .
$$

A direct estimate of the correlation coefficient to be expected in the crosscorrelation analysis of the reconstructed objects is given by $Q^{2}$.

The parametric study shows that the accuracy of the tomographic reconstruction is largely determined by two experimental parameters: the particle 
image density (in particles per pixel, ppp, in the recorded images) and the number of cameras. The former is a measure of the amount of signal contained in the measurement volume and the latter expresses how many 2D views are available of the given $3 \mathrm{D}$ particle distribution. Figure 4 shows the reconstruction quality $Q$ as a function of the number of cameras and the particle image density. The results of the simulation performed in a $2 \mathrm{D}$ domain have been translated to the case of a 3D measurement taking into account the additional dimension by dividing the particle image density by the particle image diameter (3 pixels). For a seeding density of 0.05 ppp the 2-camera system proves to be largely insufficient, whereas $Q$ rapidly increases going to 3,4 and 5 cameras. The additional camera provides additional information on the object, which increases reconstruction accuracy. Conversely, for a fixed number of cameras increasing the particle density produces a larger percentage of ghost particles, consequently decreasing the reconstruction quality. On the other hand, a larger number of particles allows a higher spatial sampling rate of the flow, returning a higher spatial resolution, which is usually desired. Based on the simulation results (Fig. 4) the maximum imaged particle density yielding an acceptable reconstruction quality $(Q>0.75)$ is 0.05 particles per pixel (50000 particles per megapixel) for a 4-camera system, which is rather advantageous with respect to particle-detection and -tracking procedures typically yielding 5000 to 10000 particles per megapixel [5, 13].

Furthermore, background light and image noise have a strong impact on the reconstruction, because the background light will be reconstructed inside the measurement volume affecting the accuracy of the particle pattern. As a result, the reconstruction quality $Q$ strongly deteriorates with increasing random image noise. However, image preprocessing operations, such as background subtraction, can be applied to reduce the mentioned effects. Furthermore, intensity normalization and the convolution with a $3 \times 3$ kernel with Gaussian distribution are commonly applied in real experiments to account for differences in sensitivity between cameras and to further reduce uncorrelated pixel noise.

A 3D numerical simulation of a vortex ring flow has been performed to validate the procedure and to assess the velocity measurement accuracy. The $35 \times 35 \times 7 \mathrm{~mm}^{3}$ measurement volume contains 24500 tracer particles, which are imaged onto four $700 \times 700$ pixel cameras resulting in a particle image density of $0.05 \mathrm{ppp}$. The maximum particle displacement between the two exposures is 2.9 voxels. The volume is reconstructed at $700 \times 700 \times 140$ voxels resolution returning a reconstruction quality factor $Q$ of 0.75 as predicted in Fig. 4. The measured displacement field contains $66 \times 66 \times 10$ vectors, which are obtained from a crosscorrelation analysis of the reconstructed volumes with $41^{3}$ interrogation volumes at $75 \%$ overlap. The vector field is shown in Fig. 5, left, where the overall motion is well captured. Furthermore, the isovorticity surface shows the expected torus shape.

The crosscorrelation coefficient $(>0.56)$ and the average signal-to-noise ratio (first-to-second highest peak) approaching 5 indicate a high confidence 

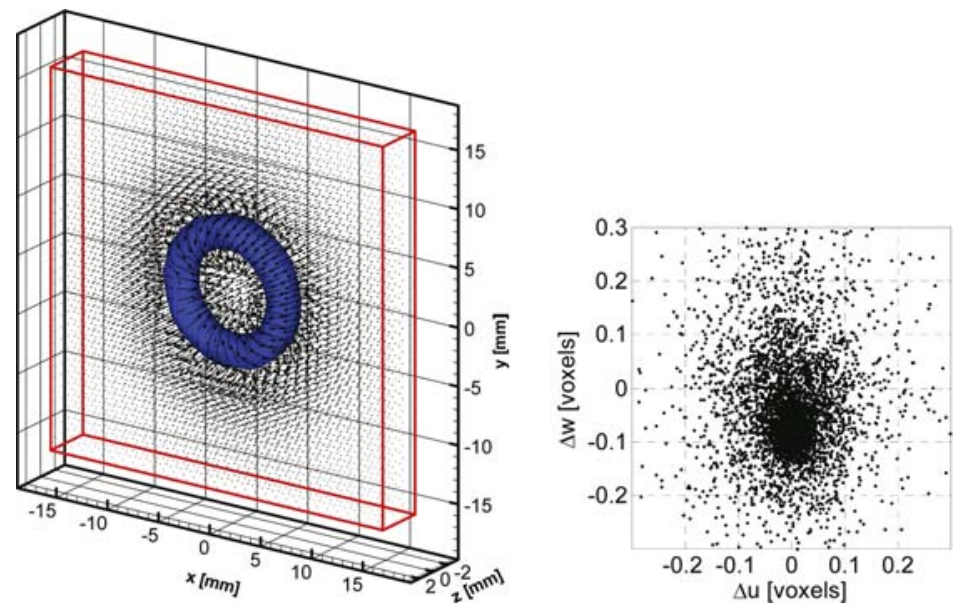

Fig. 5. Velocity field and isovorticity surface ( 0.13 voxels/voxel) from the simulated vortex ring (left) and scatter plot of the measurement error for the $u$-component and depth component $(w)$ of the particle displacement (right)

level for the measurement. The final measurement accuracy is presented in a scatter plot of the error in the $u$ - and $w$-component of the particle displacement (Fig. 5, right), where $90 \%$ of the vectors have an absolute error smaller than 0.10 voxels for the $u$ - and $v$-component and less than 0.16 voxels for the $w$-component. The larger uncertainty in depth is ascribed to the viewing configuration with angles of $30^{\circ}$ wrt the $z$-axis, which agrees with former studies on stereoscopic PIV. Furthermore, the asymmetry on the $w$ component should not be interpreted as a bias error resulting from the tomographic reconstruction, because it also appears when the exact light distribution $E_{0}(X, Y, Z)$ is crosscorrelated and results from the combination of the limited spatial resolution (modulation error) and the asymmetric distribution of the $w$-component within the measured volume.

\section{Applications to Circular Cylinder Wakes}

\subsection{Experimental Procedure}

The first application of the tomographic-PIV technique has been performed measuring the cylinder wake flow in a low-speed open-jet wind tunnel of Delft University of Technology $[6,12,14]$. The wind tunnel has a $40 \times 40 \mathrm{~cm}^{2}$ square crosssection in which a circular cylinder of $8 \mathrm{~mm}$ diameter is placed horizontally or vertically (Fig. 6). The free-stream velocity is $5 \mathrm{~m} / \mathrm{s}$ with a corresponding Reynolds number of 2700 based on the cylinder diameter.

The flow is seeded with $1-\mu \mathrm{m}$ droplets at a concentration resulting in a particle image density in the range of $0.02-0.08 \mathrm{ppp}$. The illumination source 
is a Quanta Ray double-cavity Nd:YAG laser from Spectra-Physics with a pulse energy of $400 \mathrm{~mJ}$. A slit is added in the path of the laser lightsheet cutting the low-intensity side lobes from the light profile to obtain a sharply defined illuminated volume of $8 \mathrm{~mm}$ thickness. The time separation between exposures is $35 \mu \mathrm{s}$, yielding a free-stream particle displacement of $0.18 \mathrm{~mm}$ corresponding to 3.2 voxels. The particle images are recorded at 18.3 pixels/mm resolution using 4 CCD cameras (PCO Sensicam QE and LaVision Imager Intense) with $1376 \times 1040$ pixels and 12-bit quantization. The views are arranged from the vertices of a rectangle as shown in Fig. 6 . The viewing directions with respect to the cylinder axis ( $z$-direction) are $\pm 22^{\circ}$ in the vertical direction and $-10^{\circ}$ and $+20^{\circ}$ in the horizontal directions. The cameras are equipped with Nikon lenses using $f / 8$ and Scheimpflug adapters to have the entire volume within the depth-of-focus. The imaging system is calibrated by recording images of a plate with $15 \times 12$ marks (crosses) at three depth positions separated by $4 \mathrm{~mm}$. At each calibration $Z$-location the relation between the physical coordinates $(X, Y)$ and image coordinates is described by a 3rd-order polynomial fit with an accuracy of approximately 0.2 pixels. Linear interpolation is used at intermediate $Z$-locations.

The reconstruction process is improved by means of image preprocessing with background-intensity removal, particle-intensity equalization and a Gaussian smooth $(3 \times 3$ kernel size $)$, as described in the previous section.

The intensity distribution in the measurement volume is reconstructed at 18.2 voxels $/ \mathrm{mm}$ resolution using the MART algorithm with 5 iterations. The dimensions of the reconstructed volume are 36.5 (length) $\times 35.8$ (height) $\times$ 11 (depth) $\mathrm{mm}$ corresponding to $667 \times 654 \times 203$ voxels. Note that the reconstructed volume depth is slightly larger than the lightsheet thickness to ensure that the complete lightsheet is contained, which is important for accurate reconstruction. Figure 7 shows an example of a reconstructed volume. The lightsheet position can be clearly identified within the reconstructed volume due to the application of a slit in the light path. Further analysis of the reconstructed volume yields an estimated ratio of actual particles over ghost particles of 2 for a particle image density of $0.05 \mathrm{ppp}$ [12].

The particle displacement is obtained using a 3D FFT-based crosscorrelation algorithm with iterative multigrid and window deformation [8]. The analysis returned $77 \times 79 \times 15$ velocity vectors using an interrogation volume size of $31^{3}$ voxels with $75 \%$ overlap. Data validation based on a signal-to-noise ratio threshold of 1.2 and on the normalized median test with a maximum threshold of $2[15]$ returns $5 \%$ spurious vectors. The average signal-to-noise ratio and normalized correlation coefficient are 3.3 and 0.6 , respectively, for a particle image density of $0.05 \mathrm{ppp}$.

\subsection{Results}

An instantaneous velocity and vorticity field is shown in Fig. 8, left. The separated shear layer is visible in the form of a vorticity sheet emanating from 


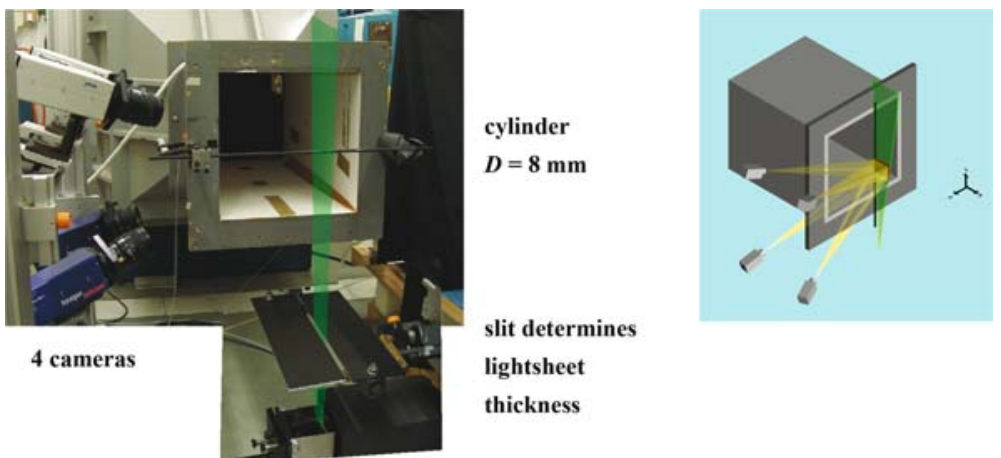

Fig. 6. The optical arrangement for the tomographic-PIV experiments in the low-speed wind tunnel. Left: horizontal-cylinder experiments. Right: schematic of vertical-cylinder experiments

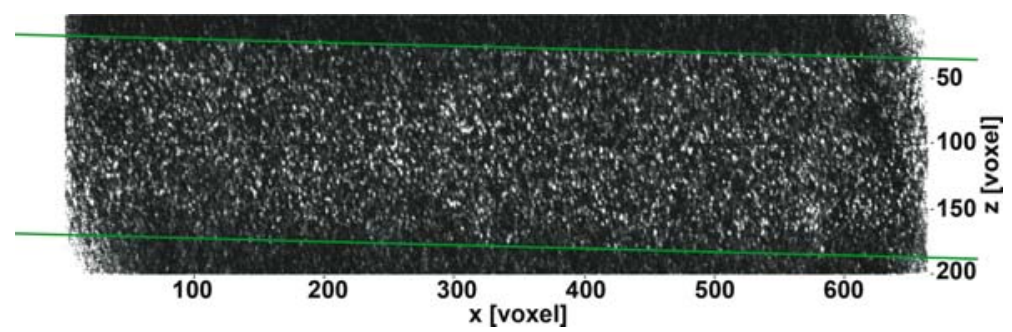

Fig. 7. Top view of the reconstructed volume showing the light intensity integrated in the $y$-direction. The green lines indicate the position of the lightsheet

the upper and lower sides of the cylinder. On the bottom side of the wake a counterclockwise roller is being formed as also indicated by the swirling pattern of the instantaneous velocity vectors. The previously shed primary roller can be identified with the roll-up of the vorticity sheet on the upper side just downstream of the first primary vortex. Finally, a third Kármán vortex with the same rotation sign as the first one is visible downstream at the bottom of the measurement volume. Even in this relatively thin view of the cylinder wake a significant three-dimensional behavior can be observed with a secondary roller interconnecting the second and the third vortex oriented approximately at $45^{\circ}$ and exhibiting a vorticity level comparable with the primary rollers. At the present Reynolds number the shear layers separating from the cylinder are transitional and three-dimensionality on the scale of the Kármán vortices is expected [16]. The isosurfaces of vorticity stretching vector magnitude $(|\vec{\omega} \cdot \overline{\overline{\nabla V}}|$, Fig. 8, right) show that the stretching activity is concentrated in the core of the secondary roller and indicates that these structures are responsible for the increase and reorientation of the vorticity between the main rollers. 


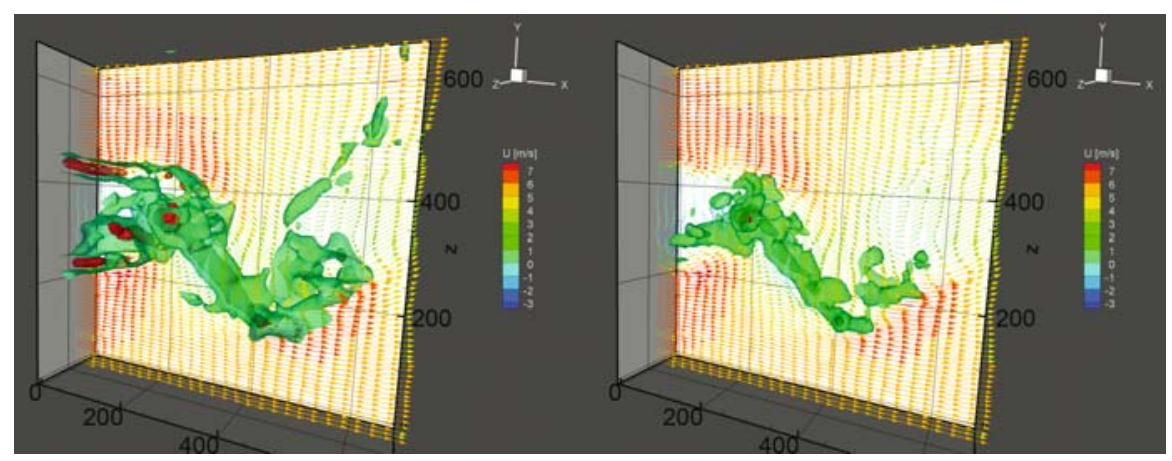

Fig. 8. Instantaneous flow-field snapshot. Left: vorticity vector magnitude isosurfaces $\left(|\omega|=2 \times 10^{3} \mathrm{~s}^{-1}\right.$, green; $|\omega|=4 \times 10^{3} \mathrm{~s}^{-1}$, red $)$ and velocity vectors in the midsection of the measurement volume. Vectors color code the streamwise velocity component. Right: vorticity stretching vector magnitude isosurfaces $(|\vec{\omega} \cdot \overline{\overline{\nabla V}}|=$ $5 \times 10^{6} \mathrm{~s}^{-2}$, green $|\vec{\omega} \cdot \overline{\overline{\nabla V}}|=15 \times 10^{6} \mathrm{~s}^{-2}$, red $)$

To improve the visualization of the structural organization of the flow the spanwise and the combination of streamwise and $y$-component of vorticity are color-coded as shown in Fig. 9. The four uncorrelated snapshots show consecutive phases of the vortex-shedding cycle. The transition from snapshot (a) to (b) marks the separation of a Kármán vortex from the upper shear layer with stretching of the secondary vortex between this Kármán vortex and the new vortex forming from the lower shear layer. This observation is consistent with the large value for the vorticity stretching magnitude at this location in Fig. 8, right, which is computed from snapshot (a). The snapshots (c) and (d) show further stretching and curling of the secondary vortices around the Kármán vortex as it is convected to $x / D=4.2$ and 4.4, respectively. Moreover, the normalized vorticity level in the Kármán vortices $\omega_{z} D / u_{\infty}=2.2$ agrees closely with planar PIV measurements in the Reynolds number range 2000 to 10000 from [17], who report an average normalized peak vorticity of 2.1 at $x / D=3$.

The spanwise organization of the flow structure becomes more visible from the vertical-cylinder experiments (Fig. 10). The secondary vortex structures (blue and red depending on the orientation of vorticity in the streamwise direction) appear to be organized in counterrotating pairs yielding a quasiperiodic behavior. From a visual inspection of 100 snapshots the normalized spatial wavelength $\lambda_{z} / D$ is estimated at 1.2 [14], which is in good agreement with [17] and slightly in excess of the reported values at lower Reynolds number [16]. The effect of the secondary structures is to first distort the primary Kármán vortices, as clearly seen in Fig. 10 , right at $x / D=4.5$, and finally cause breakup of the primary vortices. 


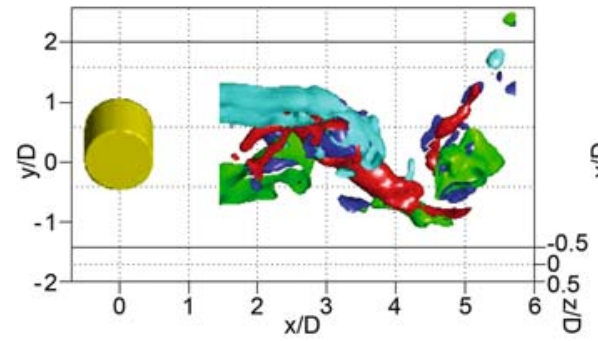

(a)

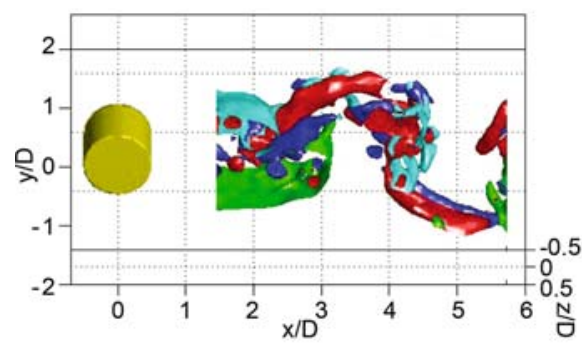

(c)

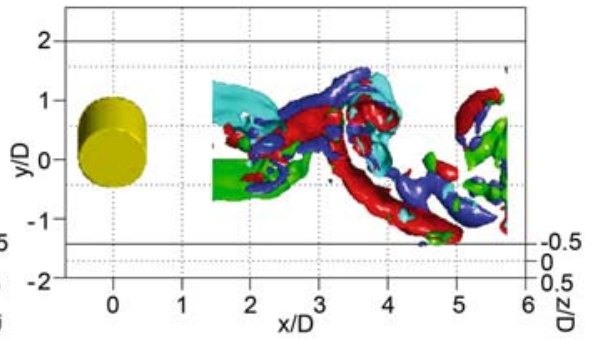

(b)

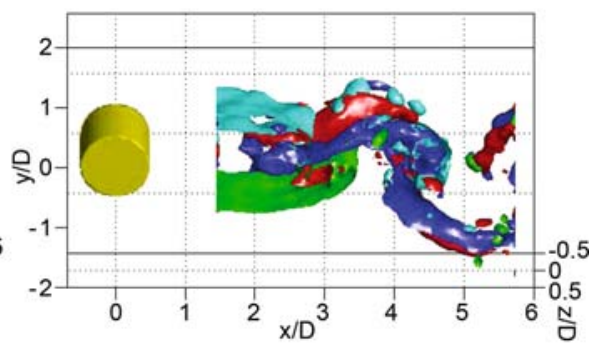

(d)

Fig. 9. Instantaneous vorticity isosurfaces showing four consecutive phases of the vortex shedding cycle $\left(\omega=1.4 \times 10^{3} \mathrm{~s}^{-1}\right.$. Color coding: cyan $\omega_{z}<0$; green $\omega_{z}>0$; blue $\sqrt{\omega_{x}^{2}+\omega_{y}^{2}} \cdot \operatorname{sign}\left(\omega_{x}\right)<0 ;$ red $\sqrt{\omega_{x}^{2}+\omega_{y}^{2}} \cdot \operatorname{sign}\left(\omega_{x}\right)>0$

The returned spatial organization of the flow and vorticity level compare well with what is reported in the literature, as shown above, so that the current results demonstrate that the instantaneous structures in the flow can be captured in a $3 \mathrm{D}$ volume with tomographic PIV.

\subsection{An Experimental Assessment: Comparison with Stereo-PIV}

In the same experimental configuration the accuracy of the tomographic-PIV technique is investigated by comparing the flow statistics with measurements made with stereoscopic PIV. The ensemble for both techniques consists of 100 snapshots obtained in the horizontal-cylinder configuration (Fig. 6, left). The limited ensemble size is due to the fact that tomographic reconstruction and interrogation operations are time consuming. Stereo-PIV results are obtained using two cameras from the above imaging system and a 2-mm thick lightsheet. Self-calibration on the particle images [11] is applied to eliminate lightsheet misalignment errors in stereo-PIV. To compare the results, vertical profiles (at $x / D=2.6$ ) of the mean and RMS fluctuations of the streamwise velocity component are extracted from the data (Fig. 11). The overall agreement between stereo- and tomographic PIV is good, with the largest 

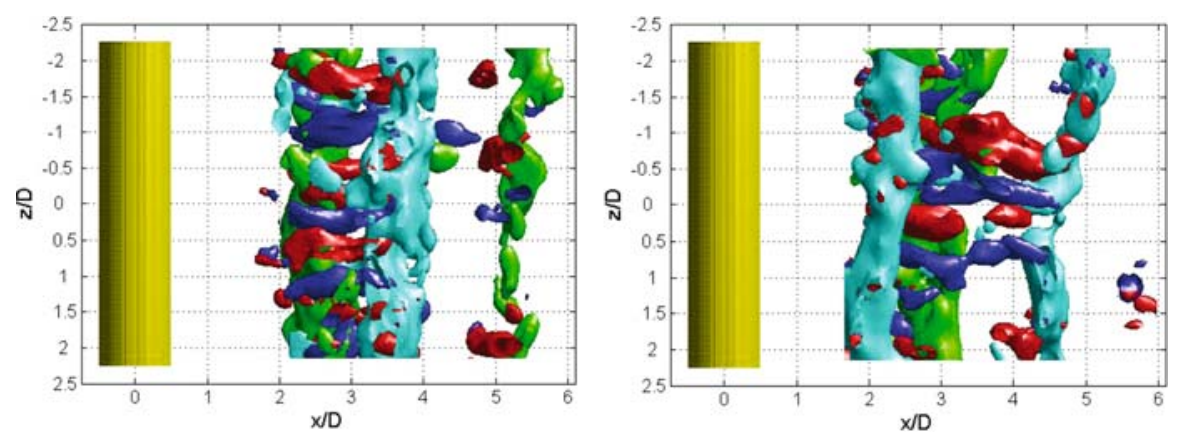

Fig. 10. Instantaneous vorticity isosurfaces from vertical-cylinder experiments. Color coding as in Fig. 9
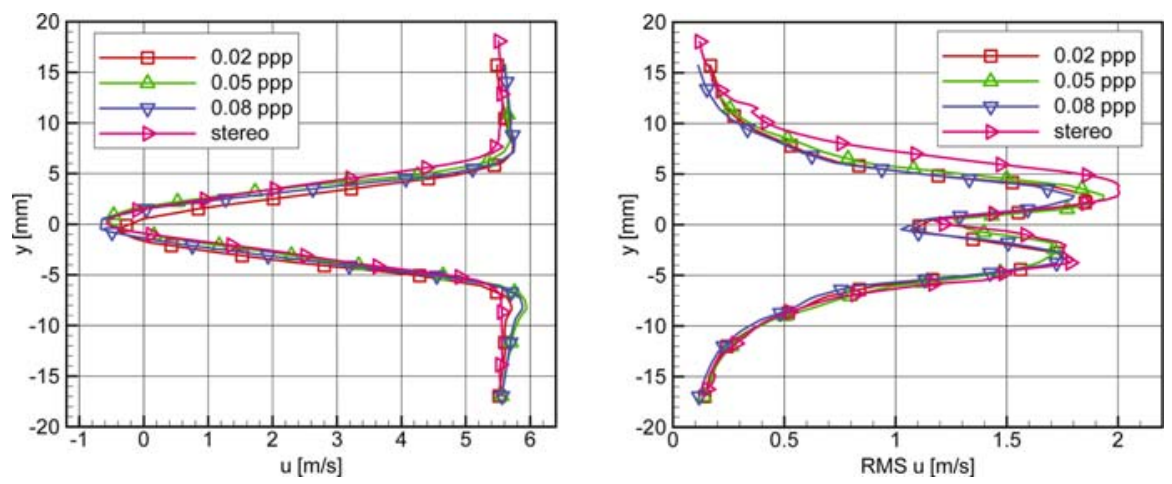

Fig. 11. Profiles of the $u$-component of velocity at $x / D=2.6$, mean (left) and RMS (right). Comparing tomographic-PIV at different seeding densities with stereo-PIV

difference in the mean $u$ of $0.50 \mathrm{~m} / \mathrm{s}$ (corresponding to 0.30 voxel particle displacement). The RMS profiles for the $u$-component agree within $0.30 \mathrm{~m} / \mathrm{s}$. Wieneke and Taylor [18] confirmed this level of agreement based on measurements of a vortex ring. It is interesting to observe that the tomographic results show very little variations with respect to the particle image density in the range of 0.02 to $0.08 \mathrm{ppp}$ for the present experiment. Further details of this experimental assessment are given in [12].

\subsection{Time-Resolved Measurements in Water Flow}

The dynamics of the primary and secondary vortices in the cylinder wake are investigated further by means of time-resolved tomographic-PIV experiments, which were carried out in a water flow at several Re $_{\mathrm{D}}$ ranging between 180 
and 2520 with $D=12 \mathrm{~mm}$. A more detailed description of the experiments can be found in [19]. The cylinder axis was aligned with the 16-mm thick lightsheet as in the previous section (Fig. 6, right). Due to the low velocity in water (of the order of a few $\mathrm{cm} / \mathrm{s}$ ) a recording rate of 10 image pairs/s proved to be sufficient to follow the time evolution of the large scales in the flow. The 30- $\mu \mathrm{m}$ latex particles were illuminated with a Quantel CFR-200 laser providing pulses of $200 \mathrm{~mJ}$ at a rate up to $30 \mathrm{~Hz}$. Four high-resolution CCD cameras (Imager Pro X, $2048 \times 2048$ pixels, 14-bit) were used to record the particle images. The crosscorrelation analysis with $48^{3}$ voxel interrogation volumes at $75 \%$ overlap returned $174 \times 117 \times 32$ vectors in the $88(\mathrm{~L}) \times 59(\mathrm{H}) \times$ 16(D) $\mathrm{mm}^{3}$ measurement volume.

A sample of the time evolution of the vortical flow structures is shown in Fig. 12 for the case of $\operatorname{Re}_{\mathrm{D}}=540$. Six snapshots separated by $200 \mathrm{~ms}$ ( $15 \%$ of the shedding period) each are presented. The vorticity magnitude in the secondary flow structures is comparable with or higher than that of the Kármán vortices, which are no longer visible downstream of $x / D=4$ at the present level of display. The secondary vortices, however, show a significant persistence even up to the edge of the observable domain, as shown by the evolution of three of those structures in Fig. 12 (indicated by the arrows in the first and last snapshot). The streamwise coherence length of the secondary vortices locally exceeds the distance between the main rollers, indicating a 3D organization that can be assimilated to Mode B [16]. However, some uncontrolled disturbances in the free stream do not allow us to draw conclusions about the flow statistical properties.

\section{Application to Turbulent Boundary Layers}

\subsection{Coherent Motion}

A fully developed turbulent boundary layer at $\operatorname{Re}_{\theta}=1900\left(\delta_{99}=24 \mathrm{~mm}\right.$ and $9.7 \mathrm{~m} / \mathrm{s}$ free-stream velocity) was investigated with the objective of inferring the $3 \mathrm{D}$ spatial organization of the flow along its entire height. A complete description of the results is given by [20]. The boundary layer develops along the bottom wall of the low-speed tunnel, where the point of transition is fixed by a tripping wire placed $1 \mathrm{~m}$ upstream of the measurement area.

The measurement of coherent motion in a turbulent boundary layer is a challenging task, because it requires a high dynamic range and good spatial resolution in order to resolve the velocity fluctuations. Moreover, the measurement in the proximity of solid interfaces poses several technical problems associated with stray light reflection. While the RMS fluctuations in the cylinder wake are of the order of the free-stream velocity, in the boundary layer they reduce to approximately $5 \%$ of the mean velocity. Hence, the time separation between the laser pulses was set at $100 \mu \mathrm{s}$, yielding a 26-voxel particle displacement in the free stream. The resolution in the measurement 

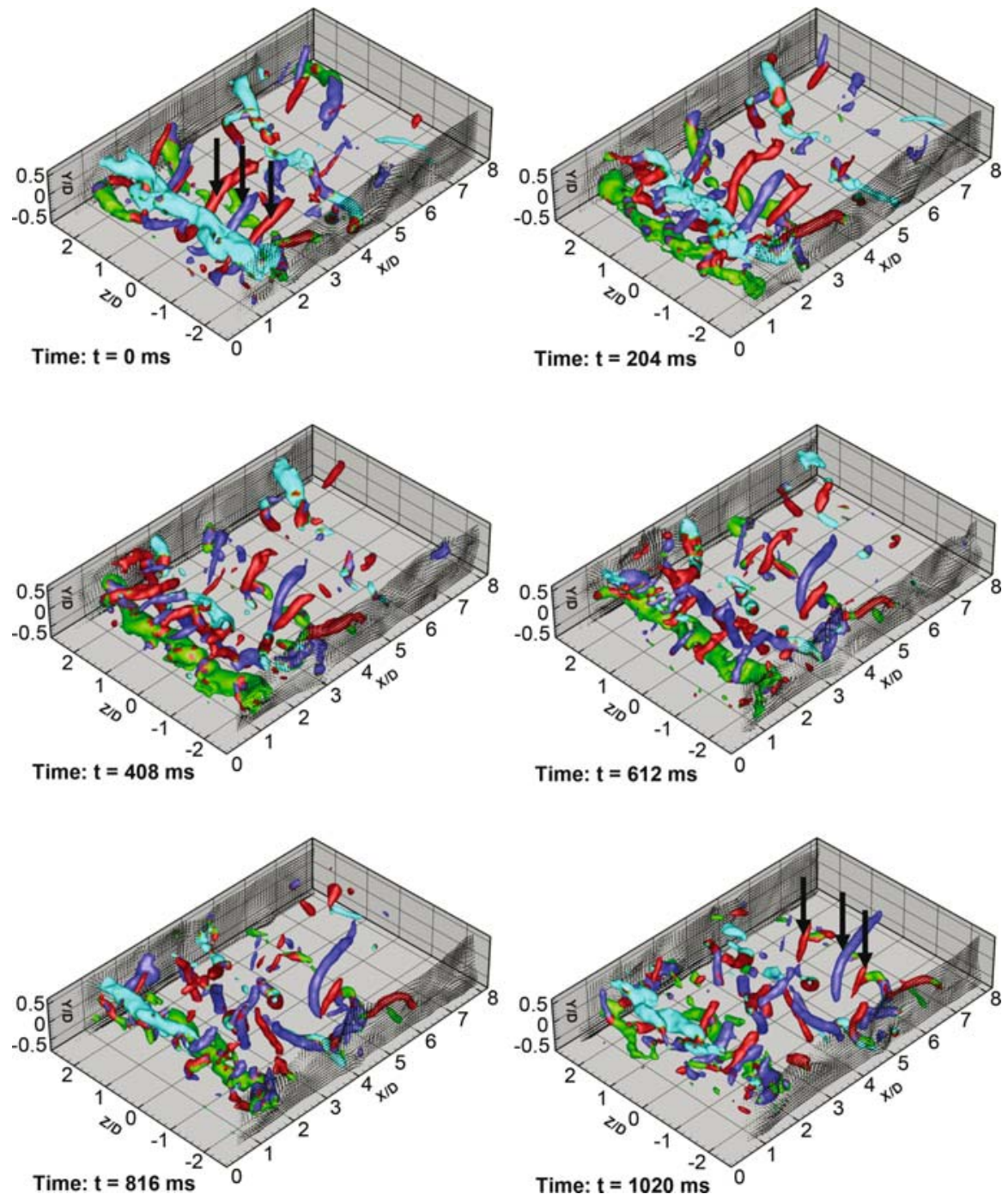

Fig. 12. Time series at $\operatorname{Re}_{\mathrm{D}}=540$ showing vortical structures detected using the $\lambda_{2}$-criterion. Color coding depending on the dominant component: when $\omega_{z}$ is dominant the color is cyan $\left(\omega_{z}>0\right)$ or green $\left(\omega_{z}<0\right)$; when $\sqrt{\omega_{x}^{2}+\omega_{y}^{2}}$ is dominant it is blue or red depending on the sign of $\omega_{x}$ 

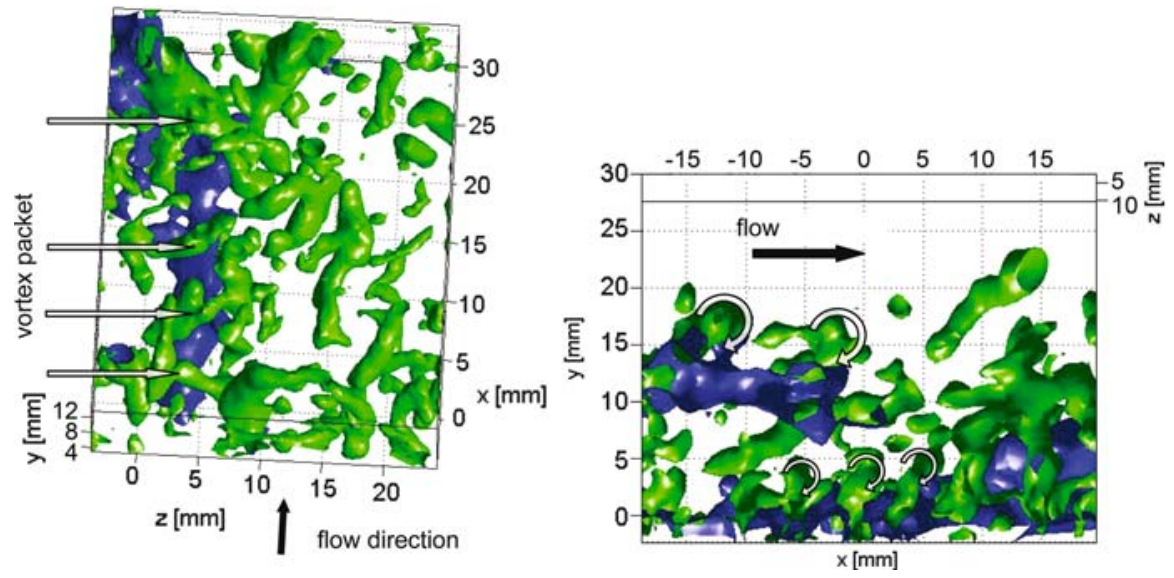

Fig. 13. Instantaneous vortex structures (Q-criterion) in green and low-speed regions in blue ( $u<85 \%$ of local mean) in a volume parallel to the wall (left) and normal to the wall (right)

volume was 26.6 voxels $/ \mathrm{mm}$ resulting in a vector spacing of $0.47 \mathrm{~mm}$ (applying $50^{3}$ crosscorrelation volumes at $75 \%$ overlap).

Two examples of the measured spatial organization of the vortex structures and low-speed regions are presented in Fig. 13. Vortical structures are identified by means of the Q-criterion [21], whereas low-speed streaks are defined with a threshold of $u<85 \%$ of local mean velocity. Figure 13, left, shows a measurement volume with the largest dimensions parallel to the wall where the $x$-axis corresponds to the streamwise direction, $y$ is the distance from the wall and $z$ is the spanwise direction. A range of vortex structures can be observed such as streamwise vortices (e.g., $5<x<20 \mathrm{~mm}, z=15 \mathrm{~mm}$ ) and arch cane or hairpin vortices, which can be both symmetrical (e.g., $x=0 \mathrm{~mm}$, $10<z<17 \mathrm{~mm}$ ) or asymmetrical (e.g., $x=17 \mathrm{~mm}, z=12 \mathrm{~mm}$ ). Furthermore, a series of four hairpin-like vortices aligned in the streamwise direction with a low-speed region between their legs is observed at $z=4 \mathrm{~mm}$, which may be recognized as a hairpin packet as introduced by [22]. The length of this packet is $25 \mathrm{~mm}$ or $1.3 \delta$ and its width is approximately $5 \mathrm{~mm}$, corresponding to 100 viscous length scales. More hairpin packets are shown in Fig. 13, right, where the measurement volume depth is oriented in the spanwise direction $(z)$ such that the whole boundary-layer height is included in the volume. Also in this case, a packet of three hairpin vortices is observed close to the wall. Arrows indicate the orientation of vorticity in the hairpin heads, which are separated in the streamwise direction by approximately 100 viscous length scales. Downstream of the packet and shifted in the $z$-direction more hairpin structures are detected, however, it is not evident whether they also belong to or interact with the packet upstream. Further from the wall at $y=15 \mathrm{~mm}$ 
two hairpins (indicated by the arrows) seem to form another packet. Away from the wall both the size of the individual vortices and their spacing in the streamwise direction increase as expected.

The statistical relevance of each of the above structures remains to be explored, but the presented results show that these larger structures within the boundary layer can be observed directly without looking at their signatures as in planar and point methods, which is an important advantage of 3D PIV methods in turbulence research.

\subsection{Time-Resolved Measurements of a Turbulent Boundary Layer and Spot in Air}

In a recent feasibility study [23] tomographic PIV has been applied to timeresolved PIV recordings of a turbulent spot and a turbulent boundary layer in air. The turbulent spot is generated in a laminar boundary layer flow over a flat glass plate with elliptical leading edge in the 1-m wind tunnel of DLR Göttingen at a free-stream velocity of $7 \mathrm{~m} / \mathrm{s}$ with zero pressure gradient (Fig. 14, right). With a short 1-ms local flow injection at $\left(\operatorname{Re}_{x}\right)^{0.5}=300$ an intense initial disturbance is introduced into the laminar boundary layer resulting in the growth of a turbulent spot, which is measured downstream at $\left(\operatorname{Re}_{x}\right)^{0.5}=450$ in a $34(\mathrm{~L}) \times 30(\mathrm{H}) \times 19(\mathrm{D}) \mathrm{mm}^{3}$ volume. The turbulent boundary layer is generated by a series of tripping wires

The experimental arrangement and procedure are similar to the experiments presented above with two important modifications. First, a high repetition rate PIV hardware is used, which consists of four Photron APX-RS CMOS cameras and a high repetition rate $\mathrm{Nd}$ :YAG laser from Lee Laser Inc with a pulse energy of $21 \mathrm{~mJ}$ at $5 \mathrm{kHz}$. The high-speed system is capable of recording $800 \times 768$ pixel images at $5 \mathrm{kHz}$ without frame straddling. Secondly, the light scattered by the $1.5-\mu \mathrm{m}$ oil particles is increased by adding two coated and highly reflective dielectric mirrors to the laser optics reflecting the light back and forth through the test section (see sketch in Fig. 14, left), which effectively increased the laser pulse energy from $21 \mathrm{~mJ}$ to an equivalent of $150 \mathrm{~mJ}$. The mirrors are placed on both spanwise sides outside the free stream so as not to disturb the flow. Optimization of the particle illumination is crucial under the present conditions, since laser power and camera sensitivity are limited, while the illuminated region is thick.

The 4D data with approximately 48000 instantaneous velocity vectors for each volume measured with $5 \mathrm{kHz}$ and $4 \mathrm{kHz}$ provides all main flow structure information, which has often been desired in former investigations of wallbounded turbulent flows. The number of spurious vectors is less than $3 \%$ for the considered cases.

Four instantaneous velocity fluctuation vector volumes separated in time by $500 \mu$ s each are represented in Fig. 15 by 3D isovorticity contour surfaces and two $3 \mathrm{C}$-velocity vector fields in $x y$-planes with $2.5 \mathrm{~mm}$ distance. The shown vectors are with respect to the nonconverged average velocity profile 

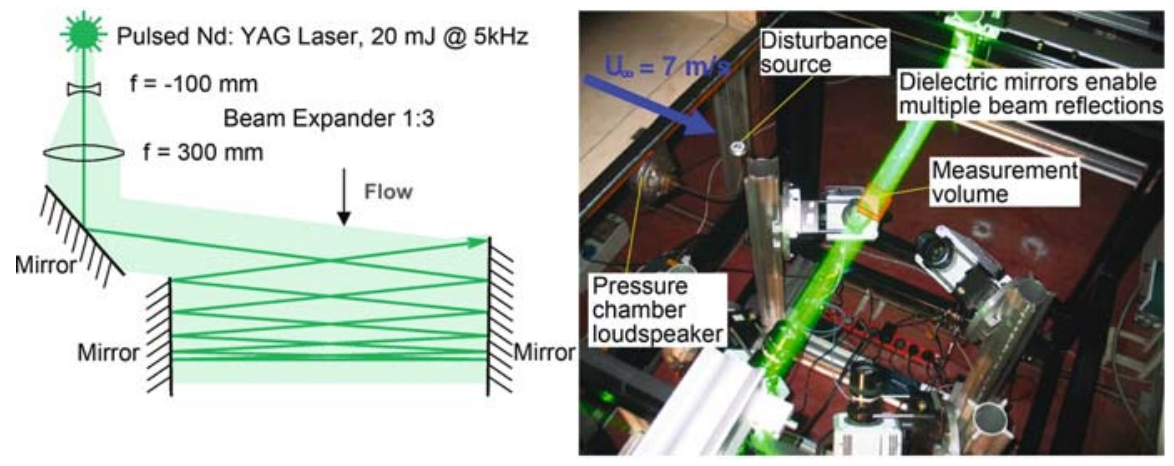

Fig. 14. Sketch of the optical setup that enables the optimal usage of the laser pulse energy of only $21 \mathrm{~mJ}$ per pulse for illumination of the PIV measurement volume (left). Experimental arrangement (right). The 4 high-speed cameras are located underneath the glass plate
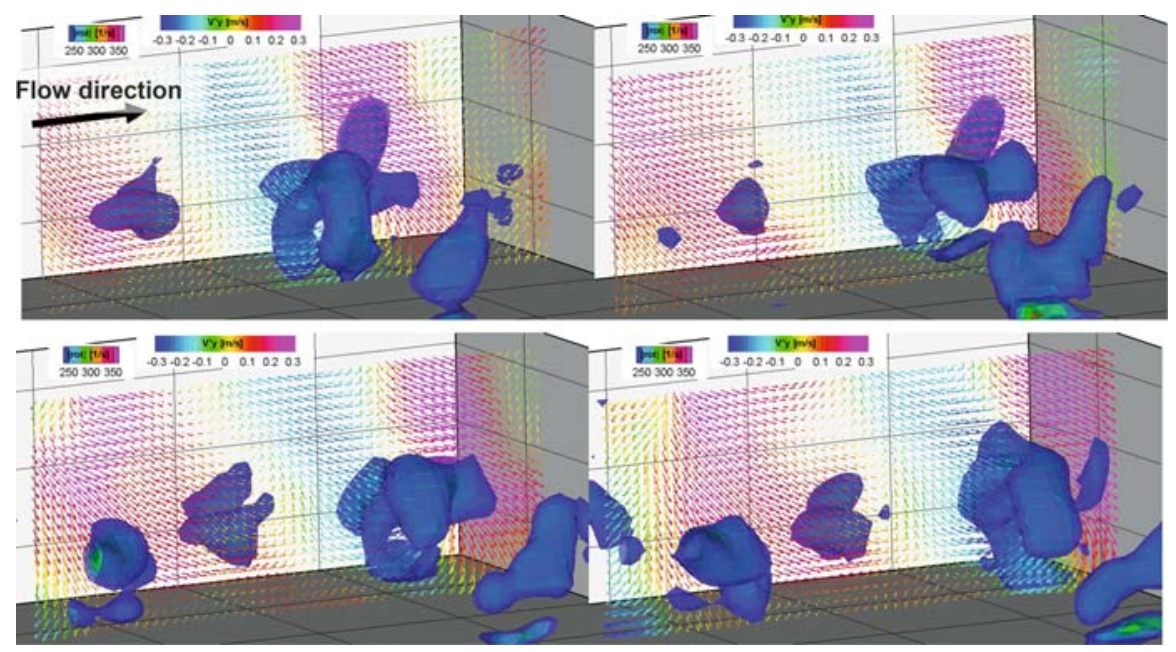

Fig. 15. Time series of instantaneous isovorticity surfaces and two $x y$-planes of $3 \mathrm{C}$ fluctuation velocity vector fields with $t=500 \mu \mathrm{s}$ shift each in a tripped turbulent boundary layer 
over only 70 volumes. Arch or hairpin structures with negative relative velocities $u^{\prime}$ are found with a strong Q2 event $\left(u^{\prime}<0 ; v^{\prime}>0\right)$ region located directly upstream of this vortex structure and a Q4 event $\left(u^{\prime}>0 ; v^{\prime}<0\right)$ region located directly downstream. Following the flow development in time the Q4 event downstream of the mentioned arch turns into a Q3 event $\left(u^{\prime}<0\right.$; $\left.v^{\prime}<0\right)$ close to the wall, which guide the flow in between the arch into an upstream Q2 event. This Q2 event influences the upstream flow so that a deflected Q4 event leads to another young arch structure following the path of the downstream one. Here, almost the same velocity distribution can be found up- and downstream of this structure.

In Fig. 16 a series of three velocity vector volumes with a time difference of $400 \mu \mathrm{s}$ in between demonstrates the explosive growth of a Q2 event and related arch or hairpin-like structure at the trailing edge (TE) of the turbulent spot. The preprocess for the rapid growth of this new turbulence producing structure is a deflected Q4 event, which interacts with the downstream flow structures of the spot with much lower velocities. This process is visible in the related part of the vector volume (not shown here). This blockage for the Q4 flow results in a deflection and change of the relative flow direction. The Q4 event with high velocities transforms into a slightly spanwise directed Q3 event, which then hits the near-wall region. At this position only a Q2 event can help to "satisfy the continuity equation". The fluid rapidly shoots upwards against the relative flow velocity while convecting downstream and the precondition for the start of a new similar process is given. The effect of the rapid growth of Q2 events has also been shown by Schröder and Kompenhans [24] by means of multiplane stereo-PIV, but the number of time steps per measurement plane was limited to two, so that the whole complex process could not be fully described nor understood. This scenario has been recognized also at other TEs of spots in the tomographic PIV dataset and can be found in a more complex distribution also inside the center of the spot structure. Of course, the present observations are based only on a few velocity vector volumes, but the similarities found in this investigation can guide future work.

\section{Summary}

Tomographic PIV is a recently developed technique for $3 \mathrm{D}$ velocity measurements, in which the three-dimensional particle distribution within the measurement volume is reconstructed by optical tomography from particle image recordings taken simultaneously from several viewing directions. The optical arrangement for illumination and recording resembles that of stereoscopic PIV and 3D-PTV. Velocity information results from three-dimensional particle pattern crosscorrelation of two volume reconstructions obtained from subsequent exposures of the particle images. The measurement chain is therefore fully digital with the advantage of being instantaneous within a volume, 


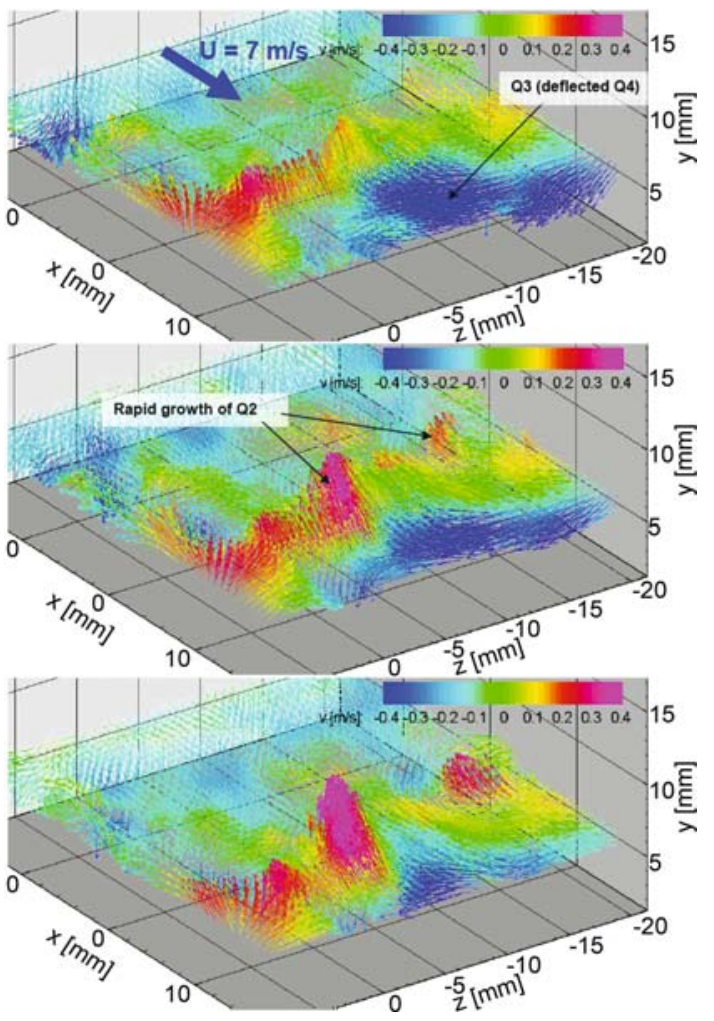

Fig. 16. Three instantaneous 3D-3C-velocity vector fields at $y=5.6,6.1$ and $6.6 \mathrm{~mm}$ of the same turbulent spot at $t=87.4$ (top), 87.8 (middle) and $88.2 \mathrm{~ms}$ (bottom) after an initial disturbance showing a rapid growth of Q2 events or hairpinlike vortices at the trailing edge $\left(u_{\mathrm{ref}}=u-6.6 \mathrm{~m} / \mathrm{s}\right)$. The colors represent the wall-normal velocity component

which makes it suitable for the analysis of flows irrespective of the flow speed. The parameters dominating the accuracy of the reconstruction procedure are the particle seeding density and the number of viewing cameras. However, the accuracy of the velocity measurement by crosscorrelation has been demonstrated not to be sensitive to the seeding density, which makes the technique particularly robust.

Using high repetition rate PIV equipment the requirements for timeresolved experiments in air flows at a moderate speed can be matched. A first application of this type was presented in Sect. 4.2 showing a turbulent boundary layer in air measured at $5 \mathrm{kHz}$. A specific illumination arrangement with multiple reflections of the light beam was needed to increase the local light intensity by a factor 7 .

The chapter presents four applications of the technique to turbulent wake flows and boundary layers in both air and water describing the experimental 
Table 1. Applications overview with some experimental parameters

\begin{tabular}{|c|c|c|c|c|}
\hline & $\begin{array}{l}\text { Cylinder } \\
\text { wake in air }\end{array}$ & $\begin{array}{l}\text { Time-resolved } \\
\text { cylinder wake } \\
\text { in water }\end{array}$ & $\begin{array}{l}\text { Turbulent } \\
\text { boundary } \\
\text { layer in air }\end{array}$ & $\begin{array}{l}\text { Time-re- } \\
\text { solved } \\
\text { boundary } \\
\text { layer in air }\end{array}$ \\
\hline $\begin{array}{l}\text { Image size } \\
\text { (pixels) }\end{array}$ & $1376 \times 1040$ & $2048 \times 2048$ & $1376 \times 1040$ & $800 \times 768$ \\
\hline $\begin{array}{l}\text { Recording rate } \\
(\mathrm{Hz})\end{array}$ & 1 & 10 & 2 & 5000 \\
\hline $\begin{array}{l}\text { Measurement } \\
\text { volume, } \\
L \times H \times D\left(\mathrm{~mm}^{3}\right)\end{array}$ & $37 \times 36 \times 8$ & $88 \times 59 \times 16$ & $33 \times 26 \times 11$ & $34 \times 30 \times 19$ \\
\hline $\begin{array}{l}\text { Spatial resolution } \\
\text { (voxels } / \mathrm{mm} \text { ) }\end{array}$ & 18.2 & 23.6 & 26.6 & 24 \\
\hline $\begin{array}{l}\text { Particle } \\
\text { concentration } \\
\left(\text { particles } / \mathrm{mm}^{3}\right) \\
\text { (total number) }\end{array}$ & $\begin{array}{l}2.1 \\
(23000)\end{array}$ & $\begin{array}{l}1.2 \\
(98000)\end{array}$ & $\begin{array}{l}3.3 \\
(32000)\end{array}$ & $\begin{array}{l}0.94 \\
(18000)\end{array}$ \\
\hline $\begin{array}{l}\text { Number of } \\
\text { vectors* } \\
\text { (total) }\end{array}$ & $\begin{array}{l}77 \times 79 \times 15 \\
(91000)\end{array}$ & $\begin{array}{l}174 \times 117 \times 32 \\
(651000)\end{array}$ & $\begin{array}{l}69 \times 54 \times 25 \\
(93000)\end{array}$ & $\begin{array}{l}46 \times 41 \times 24 \\
(45000)\end{array}$ \\
\hline
\end{tabular}

* using $75 \%$ overlap between interrogation volumes

arrangements and briefly discussing the results. All applications use a fourcamera system typically returning $50 \mathrm{k}$ to $100 \mathrm{k}$ velocity vectors using $1 k \times 1 k$ cameras or up to $650 k$ vectors using $2 k \times 2 k$ cameras. An overview of the measurement volumes, vector yields and related experimental parameters is given in Table 1. The accuracy of the velocity vector is close to that of standard PIV as assessed through synthetic experiments and by direct comparison with stereo-PIV in a real experiment.

The 3D flow organization of a Kármán wake was captured with details describing both the vortex shedding and the secondary vortex structures in the cylinder wake. A complete 3D description of hairpins and arch vortices was possible from the applications to boundary layers. Also, the interaction between low-speed regions and hairpin packets in the turbulent boundary layer was visualized. The evolution and interaction of these $3 \mathrm{D}$ structures is still not fully understood and therefore the application of 3D PIV techniques to those fluid-dynamic problems is expected to give important new insight in the future.

One of the current limitations of the technique is the processing time required for the reconstruction and interrogation (approximately $1 \mathrm{~h}$ for $10^{8}$ voxels on a Pentium $4 \mathrm{PC}$ ). Therefore, further developments of the 
tomographic-PIV technique are directed towards the reduction of the computational load by advanced algorithms for reconstruction and 3D correlation with sparse and adaptive correlation approach. Finally, the implementation of a volume self-calibration procedure will result in the improvement of the calibration accuracy.

\section{References}

[1] M. P. Arroyo, C. A. Greated: Stereoscopic particle image velocimetry, Meas. Sci. Technol. 2, 1181-1186 (1991) 104

[2] C. J. Kähler, J. Kompenhans: Fundamentals of multiple plane stereo particle image velocimetry, Exp. Fluids, Suppl. pp. S70-S77 (2000) 104

[3] Ch. Brücker: Digital-particle-image-velocimetry (DPIV) in a scanning lightsheet: 3D starting flow around a short cylinder, Exp. Fluids 19, 255-263 (1995) 104

[4] K. D. Hinsch: Holographic particle image velocimetry, Meas. Sci. Technol. 13, R61-R72 (2002) 104

[5] H. G. Maas, A. Gruen, D. Papantoniou: Particle tracking velocimetry in threedimensional flows, Exp. Fluids 15, 133-146 (1993) 104, 108, 109

[6] G. E. Elsinga, F. Scarano, B. Wienele, B. W. Van Oudheusden: Tomographic particle image velocimetry, Exp. Fluids 41, 933-947 (2006) 104, 105, 107, 110

[7] G. T. Herman, A. Lent: Iterative reconstruction algorithms, Comput. Biol. Med. 6, 273-294 (1976) 105, 106

[8] F. Scarano, M. L. Riethmuller: Advances in iterative multigrid PIV image processing, Exp. Fluids, Suppl pp. S51-S60 (2000) 105, 111

[9] R. Y. Tsai: An efficient and accurate camera calibration technique for 3D machine vision, in Proc. IEEE Conf. on Computer Vision and Pattern Recognition (1986) pp. 364-374 105

[10] S. M. Soloff, R. J. Adrian, Z.-C. Liu: Distortion compensation for generalized stereoscopic particle image velocimetry, Meas. Sci. Technol. 8, 1441-1454 (1997) 105

[11] B. Wieneke: Stereo-PIV using self-calibration on particle images, Exp. Fluids 39, 267-280 (2005) 105, 114

[12] G. E. Elsinga, B. W. Van Oudheusden, F. Scarano: Experimental assessment of tomographic-PIV accuracy, in 13th Int. Symp. on Applications of Laser Techniques to Fluid Mech (2006) paper 20.5 108, 110, 111, 115

[13] F. Pereira, H. Stüer, E. C. Graff, M. Gharib: Two-frame 3D particle tracking, Meas. Sci. Technol. 17, 1680-1692 (2006) 109

[14] F. Scarano, G. E. Elsinga, B. W. Van Oudheusden: Investigation of 3-D coherent structures in the turbulent cylinder wake using tomo-PIV, in 13th Int. Symp. on Applications of Laser Techniques to Fluid Mech (2006) paper 20.4 110,113

[15] J. Westerweel, F. Scarano: A universal detection criterion for the median test, Exp. Fluids 39, 1096-1100 (2005) 111

[16] C. H. K. Williamson: Vortex dynamics in the cylinder wake, Annu. Rev. Fluid Mech. 28, 477-539 (1996) 112, 113, 116 
[17] J. F. Huang, Y. Zhou, T. Zhou: Three-dimensional wake structure measurement using a modified PIV technique, Exp. Fluids 40, 884-896 (2006) 113

[18] B. Wieneke, S. Taylor: Fat-sheet PIV with computation of full 3D-strain tensor using tomographic reconstruction, in 13th Int. Symp. on Applications of Laser Techniques to Fluid Mech (2006) paper 13.1115

[19] D. Michaelis, C. Poelma, F. Scarano, J. Westerweel, B. Wieneke: A 3D timeresolved cylinder wake survey by tomographic PIV, in 12th Int. Symp. on Flow Visualization (2006) paper 12.1116

[20] G. E. Elsinga, D. J. Kuik, B. W. Van Oudheusden, F. Scarano: Investigation of the three-dimensional coherent structures in a turbulent boundary layer with tomographic-PIV, in 45th AIAA Aerospace Sciences Meeting, Reno, NV, USA, AIAA-2007-1305 (2007) 116

[21] J. C. R. Hunt, A. Wray, P. Moin: Eddies, stream, and convergence zones in turbulent flows, Center for turbulence research report, CTR-S88 (1988) 118

[22] R. J. Adrian, C. D. Meinhart, C. D. Tomkins: Vortex organization in the outer region of the turbulent boundary layer, J. Fluid Mech. 422, 1-54 (2000) 118

[23] A. Schröder, R. Geisler, G. E. Elsinga, F. Scarano, U. Dierksheide: Investigation of a turbulent spot using time-resolved tomographic PIV, in 13th Int Symp on Applications of Laser Techniques to Fluid Mech (2006) paper 1.4119

[24] A. Schröder, J. Kompenhans: Investigation of a turbulent spot using multiplane stereo PIV, Exp. Fluids 36, 82-90 (2004) 121

\section{Index}

ghost particle, 108, 109, 111

hairpin vortex, 118, 121

Kármán vortex, 112, 113

MART, 106, 107, 111

multigrid window deformation technique, 105

Q-criterion, 118

reconstruction quality, 109
Scheimpflug condition, 104

time-resolved PIV (TR PIV), 115, 119

tomographic reconstruction, 105

tomography, 104, 105, 121

turbulent boundary layer, 116

volume self-calibration, 124

vortex

hairpin, 118, 121

vortex ring, 109

voxel, 105 